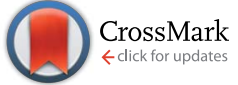

Cite this: RSC Adv., 2016, 6, 25662

\title{
Magnetite nanoparticles enable a rapid conversion of volatile fatty acids to methane $\uparrow$
}

\author{
Zhiman Yang, Rongbo Guo, ${ }^{*}$ Xiaoshuang Shi, Chuanshui Wang, Lin Wang \\ and Meng Dai
}

The objective of this work was to evaluate whether paddy soil enrichments obtained in the presence of magnetite and propionate (named SEM) can accelerate methane production from mixed VFAs. To do this, the SEM were firstly obtained via a semi-continuous subculture. The results showed that the addition of magnetite not only promoted the maximum production rate, but also reduced the methane production time in a dose-independent manner. The observation that Fe(III)-reducing Thauera was the dominant bacteria, combined with the observation that the methane production and Fe(II) formation proceeded in parallel, suggested that magnetite acted as an electron acceptor, may accelerate rapid oxidation of propionate and facilitated the establishment of a syntrophic interaction of Thauera with Methanobacterium. The SEM gave a methane yield and production rate that were on average 2.7-fold and 3.4-fold higher, respectively than those in paddy soil enrichments obtained in the absence of magnetite in conversion of the mixed VFAs to methane. The findings indicated the remarkable application potential of the SEM for improving methane production performance of the mixed VFAs.

Received 26th January 2016

Accepted 15th February 2016

DOI: $10.1039 / c 6 r a 02280 d$

www.rsc.org/advances magnetite in the process of methane production from VFAs. These observations further suggested that facilitating IET via magnetite nanoparticles might be an effective strategy to enhance the methane production rate of the mixed VFAs.

Although paddy soil enrichments obtained via the addition of magnetite and acetate showed the potential value of accelerating methane production from the mixed VFAs, ${ }^{13}$ the majority of the individual VFAs (e.g. propionate and butyrate) were not efficiently removed by this paddy soil enrichment, indicating a poor performance in the conversion of the mixed VFAs to methane. It has been reported that the supplementation of magnetite to methanogenic sludge promoted fast methanogenic propionate degradation. ${ }^{\mathbf{1 2 , 1 4}}$ Intermediates (e.g. acetate and butyrate) of propionate degradation were also detected and subsequently depleted by the enrichment. ${ }^{\mathbf{1 2}}$ These findings led to a critical hypothesis that paddy soil enrichments obtained in the presence of magnetite and propionate (named SEM) could have the capability of accelerating methane production from the mixed VFAs produced during methanogenesis. To test this hypothesis, SEM were first established via a semi-continuous subculture. The methanogenic characteristics of the SEM degrading the mixed VFAs were subsequently investigated.

\section{Materials and methods}

Shandong Industrial Engineering Laboratory of Biogas Production \& Utilization, Key Laboratory of Biofuels, Qingdao Institute of Bioenergy and Bioprocess Technology, Chinese Academy of Sciences, No. 189 Songling Road, Qingdao, Shandong Province 266101, P. R. China. E-mail: guorb@qibebt.ac.cn; Fax: +86-0532-80662708; Tel: +86-0532-80662708

† Electronic supplementary information (ESI) available. See DOI: 10.1039/c6ra02280d

\subsection{Paddy soil sample and preparation of nano-sized magnetite}

Soil samples collected from a rice field (Taizhou, China) were used as inocula. The total solid (TS) content of the paddy soil 
was $62.3 \%(\mathrm{w} / \mathrm{w})$ with $4.3 \%$ of volatile solid (VS). Magnetite nanoparticles (average $763 \mathrm{~nm}$, Fig. S1 $\dagger$ ) were synthesized as described previously by Kang et al. ${ }^{15}$ Analyses of X-ray diffraction (XRD) and Fourier transform infrared (FTIR) spectroscopy identified the resulting product as magnetite (Fig. S2 and S3†).

\subsection{Methane production}

A series of batch tests were conducted at $30{ }^{\circ} \mathrm{C}$ in $60 \mathrm{~mL}$ anaerobic bottles with a working volume of $20 \mathrm{~mL}$, except for tests 4 and 5 where $30 \mathrm{~mL}$ and $50 \mathrm{~mL}$ volumes in $120 \mathrm{~mL}$ bottles were respectively used. Each test was carried out in triplicate, and the bottles under a headspace of high purity $\mathrm{N}_{2}$ were maintained stationary in an incubator. The medium ( $\mathrm{pH}$ 7.0) used in this work was described previously. ${ }^{13}$

In test 1 , SEM were obtained via semi-continuous subcultures amended with nano-sized magnetite particles. $3 \mathrm{~g}$ of the soil sample was added to $20 \mathrm{~mL}$ of the sterile medium containing $10 \mathrm{mM}$ of propionate and $20 \mathrm{mM}$ of magnetite as $\mathrm{Fe}$ atoms. $2 \mathrm{~mL}$ of the enrichments was transferred into fresh medium supplemented with $10 \mathrm{mM}$ of propionate and $20 \mathrm{mM}$ magnetite when each run had the highest methane yield. Five generations of transfers were conducted.

In tests $2-4$, batch cultivation was performed using the resulting enrichments from the fifth generation as an inoculum at the concentration of $0.2 \mathrm{~g}$ VS per L. Test 2 was conducted to investigate the effect of the initial propionate concentrations (ranging from 10 to $100 \mathrm{mM}$ ) on methanogenesis. Test 3 was used to examine the effect of the initial magnetite concentrations (ranging from 20 to $320 \mathrm{mM}$ ) on methane production. In test 4 , the methanogenic pathway of SEM was determined via the addition of methanogenic inhibitor 2-bromoethane sulfonate (BES). The SEM were incubated in fresh medium containing $20 \mathrm{mM}$ magnetite. $100 \mathrm{mM}$ of BES was added into the medium at the initial stage of cultivation.

In test 5 for repeated batch cultivation (RBC), mixed VFAs were used which consisted of equal mmol proportions of acetate, propionate, butyrate, valerate and caproate. In the startup stage, $0.2 \mathrm{~g}$ VS per L of SEM from the fifth generation were grown in medium with $10 \mathrm{mM}$ propionate and $20 \mathrm{mM}$ magnetite. When the produced methane reached the highest yield, RBC was initiated. $20 \%(\mathrm{v} / \mathrm{v})$ of the medium was removed and the same proportion of fresh medium containing $250 \mathrm{mM}$ of the mixed VFAs was added. Methane in the headspace of bottles was removed with a vacuum pump and replaced with high purity $\mathrm{N}_{2}$ before the start of the next cycle. For each cycle of methane production, the mixed culture that had the highest methane yield was used as the inocula for the next cycle of cultivation. Three cycles of harvesting and feeding were performed.

\subsection{Analytical methods}

$\mathrm{H}_{2}$ and $\mathrm{CH}_{4}$ were periodically analyzed using gas chromatography (GC) according to the method described by Yang et al. ${ }^{16} \mathrm{In}$ brief, $\mathrm{H}_{2}$ and $\mathrm{CH}_{4}$ were determined by a gas chromatograph (SP6890, Shandong, China) equipped with a thermal conductivity detector (TCD) and a $2 \mathrm{~m}$ stainless steel column filled with
5A molecular sieves. $\mathrm{N}_{2}$ was used as the carrier gas at a flow rate of $30 \mathrm{~mL} \mathrm{~min}{ }^{-1}$. The temperatures at the injection port, oven and detector were respectively 120, 50 and $100{ }^{\circ} \mathrm{C}$. The maximum methane production rate $\left(R_{\mathrm{m}}\right)$ and the lag-phase time $(\lambda)$ were calculated according to the modified Gompertz equation. ${ }^{16}$ The methane yield was calculated by dividing cumulative methane production with the total substrate added. TS, VS, and individual VFAs were determined as described previously. ${ }^{13}$ VFAs were analyzed with a high performance liquid chromatography (HPLC) instrument (Agilent HPLC 1200 series) equipped with UV detection $(210 \mathrm{~nm})$, refractive index detection and an Aminex HPX-87P cation exchange column (Bio-Rad Laboratories, Hercules, CA) at the column temperature of 65 ${ }^{\circ} \mathrm{C} .5 \mathrm{mM}$ of $\mathrm{H}_{2} \mathrm{SO}_{4}$ was used as the mobile phase at the flow rate of $0.6 \mathrm{~mL} \mathrm{~min}^{-1}$. The XRD spectrum was analyzed with wide angle X-ray diffraction (Bruker D8 Advance) according to a conventional $2 \theta / \theta$ method. Concentrations of $\mathrm{Fe}(\mathrm{II})$ extracted with $0.5 \mathrm{~N} \mathrm{HCl}$ were determined using a ferrozine method. The total iron consisted of $\mathrm{Fe}(\mathrm{II})$ and $\mathrm{Fe}(\mathrm{III})$ and was determined after the sample was reduced with $0.25 \mathrm{M}$ hydroxylamine before the extraction of $0.5 \mathrm{~N} \mathrm{HCl}^{17}$

\subsection{DNA extraction, PCR amplification and 16S rDNA sequencing}

Samples from the fifth generation were used for total genomic DNA extraction using a CTAB/SDS method. Amplicon libraries were constructed for Illumina sequencing using bacterial primer pairs 515f/806r for the V4 region of $16 \mathrm{~S}$ rRNA. ${ }^{18}$ PCR, library construction, Illumina sequencing and sequence analysis were performed as described previously. ${ }^{13}$ The sequences were deposited to the GeneBank Sequence Read Archive with accession no. SRR1832810.

\section{Results and discussion}

\subsection{Paddy soil enrichments and their methanogenic characteristics}

A semi-continuous subculture was used to establish the SEM in this work. An area-external standard method of GC was used to calculate the methane content in all the tests in a short analysis time (Fig. S4†). Fig. 1 shows the time course of methane production over five generations of subculture. No significant improvement in the methane yield for the SEM was observed when compared with the corresponding paddy soil enrichments achieved without magnetite addition (named SEC) at the end of each generation of subculture. According to the reaction $\left(4 \mathrm{CH}_{3} \mathrm{CH}_{2} \mathrm{COOH}+2 \mathrm{H}_{2} \mathrm{O} \rightarrow 7 \mathrm{CH}_{4}+5 \mathrm{CO}_{2}\right)$, the expected methane yield for complete conversion of propionate is 1.75 $\mathrm{mol} \mathrm{CH}_{4}$ per mol propionate. The ultimate methane yield of an average of $1.6 \mathrm{~mol} \mathrm{CH}_{4}$ per mol propionate for both experimental groups was lower than the expected value. Cruz Viggi et $a .^{12}$ speculated that the low methane yield was due to the presence of alternative electron acceptors like sulfate or nitrate. However, because nitrate and sulfate were not added to the medium, and a long time of incubation might eliminate the alternative electron acceptors such as sulfate, the part of 


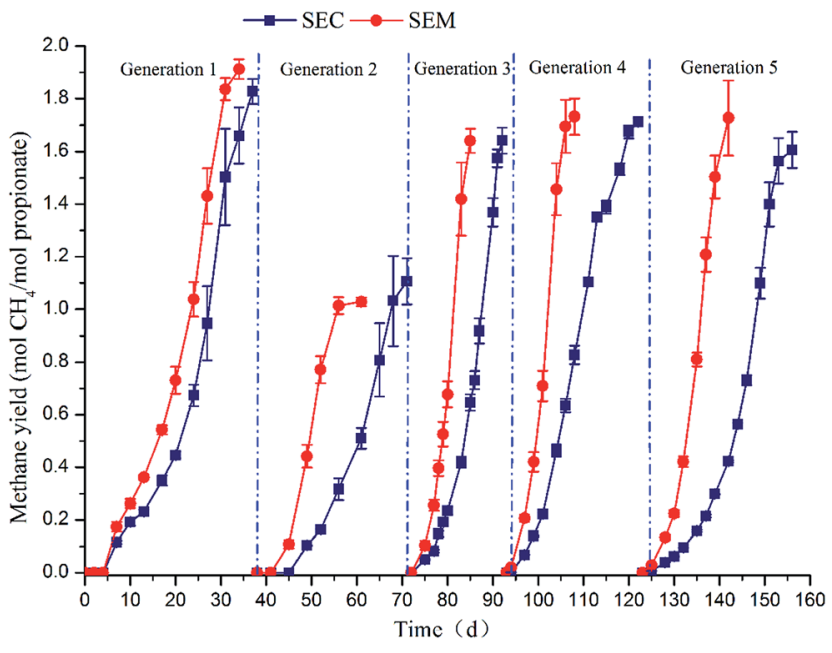

Fig. 1 Profiles of methane production in the semi-continuous enrichment subculture.

propionate consumed may be used to maintain the cell growth. Indeed, a significant amount of multi-species aggregates formed during the incubation in this work.

Fig. 1 also shows that time for conversion of propionate to methane in the SEM was much shorter than that in the corresponding SEC over each generation of subculture. Specifically, the time course in the SEM was reduced by $8.1 \%$ in generation $1,29.4 \%$ in generation $2,33.3 \%$ in generation $3,46.7 \%$ in generation 4 , and $41.2 \%$ in generation 5 compared with the corresponding SEC. As shown in Table 1, although the maximum methane rate of the SEM was similar to that of the SEC in the generation 1, a significant reduction $(31.9 \%)$ of the lag-phase time in the SEM was detected when compared with the SEC. This indicated that magnetite slightly stimulated methanogenesis at the early stage of incubation.

In four subsequent generations of subcultures, the addition of magnetite resulted in a significant decrease in the lag-phase time and a significant increase in methane production rate. For

Table 1 Methane production rate and lag-phase time in the semicontinuous enrichment subculture

$$
R_{\mathrm{m}}\left(\mathrm{mmol} \mathrm{d}^{-1}\right)
$$

SEC

Generation 1

Generation 2

Generation 3

Generation 4

Generation 5

0.019

0.012

0.026

0.020

0.018

0.018

0.023

0.042

0.048

0.032 each generation of the subcultures, the lag-phase time for methane production in the SEM was shorter than that in the SEC. On one hand, the lag-phase time in the SEM was decreased by $48.9 \%$ for generation $2,42.1 \%$ for generation $3,34.2 \%$ for generation 4 , and $56.1 \%$ for generation 5 compared with the corresponding SEC. On the other hand, the maximum methane production rate (average $0.019 \mathrm{mmol} \mathrm{d}^{-1}$ )was on average 93\% higher than that (average $0.036 \mathrm{mmol} \mathrm{d}^{-1}$ ) in the SEC. These results demonstrated that magnetite nanoparticles had the ability to accelerate methane production from propionate. In this work, magnetite added to paddy soil resulted in a significant increase in the maximum methane production rate that was higher than that of previous reports, which showed that the addition of magnetite to a methanogenic sludge resulted in a rise of up to $33 \%$ in the methane production rate from propionate. $^{\mathbf{1 2}}$

To further analyze the methanogenic characteristics of the SEM, the effects of different doses of magnetite and propionate in regulating methanogenesis were assessed. Although increasing the magnetite concentration resulted in a slight extension in lag-phase time, the methane yield and production rate were completely independent of any dose effects of the magnetite (Table S1 $\dagger$ ). This indicated that excess amounts of magnetite particles did not substantially improve methane production when the magnetite concentration was $>20 \mathrm{mM}$. It is worth noting that the methane production rate increased with the increase of propionate concentration up to $20 \mathrm{mM}$, whereas no significant improvement in methane production rate was obtained at propionate concentrations of $>20 \mathrm{mM}$. Both the methane yields and lag-phase time were also sensitive to the increase of propionate concentration, showing a decreasing trend in the methane yield as well as an extension trend in lagphase time (Table $\mathrm{S} 2 \dagger$ ). These results indicated that a high concentration of propionate might lead to an inhibition of methanogenesis. The inhibition probably resulted from the toxicity of the undissociated form of propionic acid to the cell, inhibiting the growth and metabolism of propionate-oxidizing consortia. $^{19}$

\subsection{Intermediate metabolites}

Fig. 2 shows that acetate and $n$-butyrate were the main intermediates with a trace amount of $n$-valerate and $n$-caproate in the SEM and SEC. Acetate and butyrate accumulated at an early stage of incubation in the two groups, indicating that methanogenesis and the net accumulation of acetate and $n$-butyrate may proceed in parallel. The produced acetate and $n$-butyrate were subsequently depleted and converted to methane at the end of the incubation in the two groups. Previous reports showed that propionate can be first dismutated to acetate and butyrate after which butyrate is oxidized to acetate via $\beta$-oxidation..$^{20}$ Also, some syntrophic propionate degraders can convert propionate to acetate and $\mathrm{CO}_{2}$ via a methylmalonyl-CoA pathway. ${ }^{6}$ The accumulated acetate and butyrate in the present study indicated that these two pathways performed by different microbial species could be involved in propionate degradation. Comparative analysis further showed that the addition of 

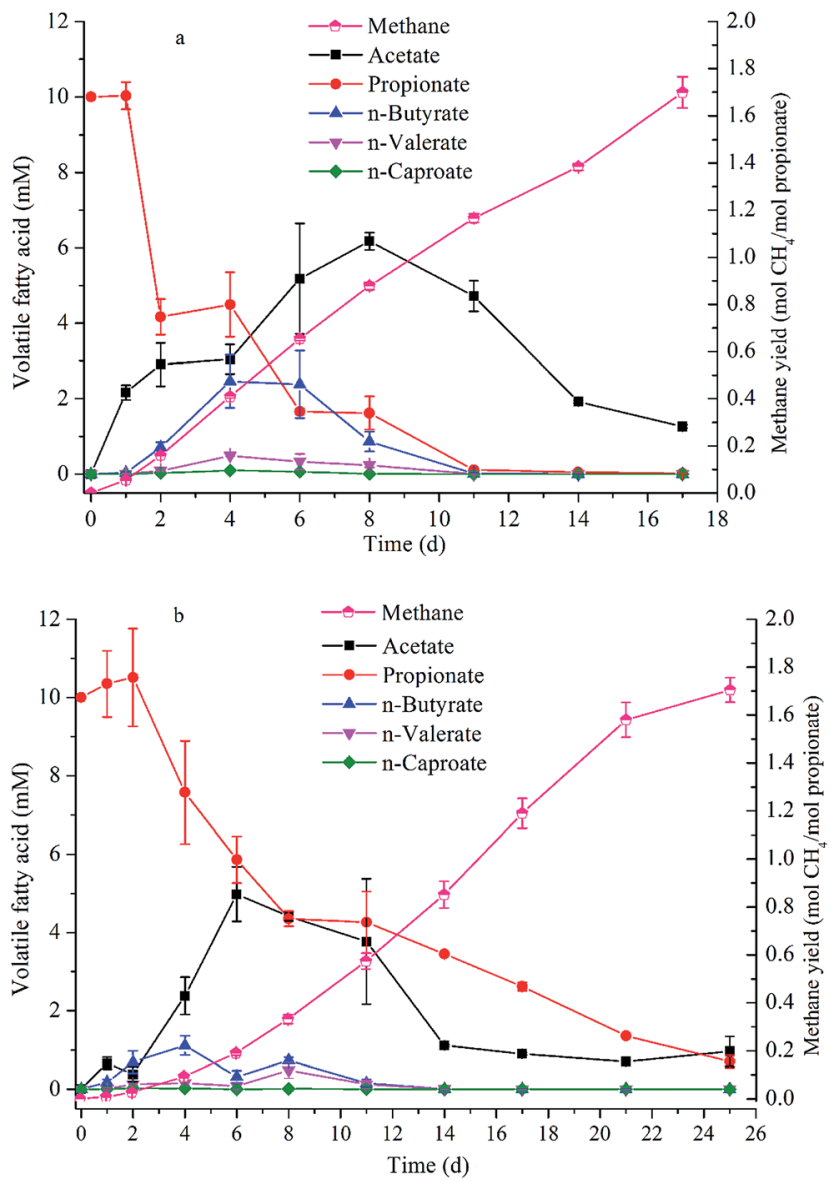

Fig. 2 The time course of the production for both methane and metabolic intermediates (a: SEM; b: SEC).

magnetite accelerated the metabolism of propionate followed by a rapid methane production. These findings, combined with the observations that magnetite enhanced the methane production rate and reduced methane production time, indicated the possibility that the SEM may promote the rates of the mixed VFA conversion to methane.

To determine the probable methane production pathways, an inhibition experiment with BES addition was carried out. Fig. S5 $\dagger$ shows that $0.8 \mu \mathrm{mol} \mathrm{H}_{2}$ per mol of propionate was produced and then consumed in the SEM group. Compared with the SEM, a significant amount of $\mathrm{H}_{2}$ was produced after $\mathrm{BES}$ addition. These findings indicated that methane was likely produced from propionate via interspecies hydrogen transfer. Interestingly, although $\mathrm{Fe}(\mathrm{II})$ concentrations over the incubation were below $0.1 \mathrm{mM}$ in both groups, results from XRD analysis showed that magnetite in both groups was transformed into $\mathrm{Fe}(\mathrm{II})_{3}\left(\mathrm{PO}_{4}\right)_{2}$ at the end of cultivation (Fig. S2 $\uparrow$ ). This, combined with the observation that significant amounts of acetate accumulated in both groups (Fig. S6 $†$ ), further suggested that Fe(III) reduction and propionate degradation occurred in parallel. According to the reaction in Table $\mathrm{S} 3, \uparrow$ the syntrophic oxidation of propionate to $\mathrm{H}_{2}$ and acetate is thermodynamically favorable. However, propionate oxidation, $\mathrm{Fe}(\mathrm{II})$ formation and methane production simultaneously proceeded in the presence of magnetite. This finding indicated that magnetite may act as an electron acceptor and accelerate propionate degradation in a similar manner to our previous report, ${ }^{21}$ which showed that magnetite facilitated methane production from ethanol by acting as an electron acceptor.

\subsection{Structure of microbial communities}

Based on the deep sequencing of 16S rRNA gene amplicons, the bacterial diversity of paddy soil enrichment from the fifth generation of subculture was defined. A small portion of archaeal sequences were amplified, using the primer pairs targeting the V4 region of $16 \mathrm{~S}$ rRNA genes for bacteria. Results from Fig. $\mathrm{S} 7$ and Table $\mathrm{S} 4 \uparrow$ show that magnetite added to paddy soil reduced the diversity of microbial communities.

The phylogenetic classification of bacteria OTUs at the genus level showed clear differences in communities between the SEM and SEC (Fig. 3). Desulfovibrio, Clostridium, Cloacibacillus, Acholeplasma, Smithella, Syntrophomonas and Dechloromonas in the SEC accounted for $1.3 \%, 4 \%, 1.1 \%, 1.4 \%, 6 \%, 4.1 \%$, and $11.9 \%$ of the total bacterial sequences, respectively, while those of the SEM were $1 \%, 2.1 \%, 0.2 \%, 0.4 \%, 2.1 \%, 0.9 \%$, and $6.9 \%$. Clostridium and Dechloromonas have been detected in acetateoxidizing environments, ${ }^{13,22}$ which, together with acetateoxidizing bacteria Desulfovibrio, ${ }^{23}$ might be related to the degradation of acetate produced in this work. Cloacibacillus is an amino-acid-degrading bacteria, ${ }^{24}$ which might degrade proteins or amino acids from the dead cells. Acholeplasma was detected in the methanogenesis from nonproductive coal, ${ }^{25}$ but its role in propionate degradation is still unknown. Smithella, Syntrophobacter and Syntrophomonas have been previously described to be related to the oxidation of propionate and butyrate in paddy soil. ${ }^{4,26} \mathrm{~A}$ higher abundance of Diaphorobacter (6\%) and Treponema (6.3\%) were observed in the SEM compared with the SEC $(4.6 \%$ and $1.1 \%)$. Diaphorobacter is described as nitrate reducing bacteria. ${ }^{27}$ Its role in propionate degradation is

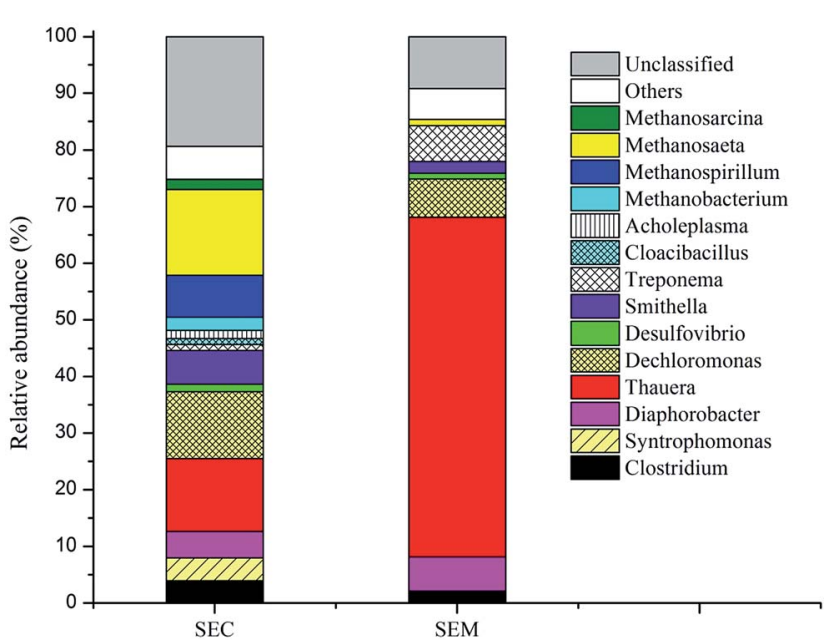

Fig. 3 Taxonomic classification of bacterial community. Genera accounting for $<1 \%$ of total composition were classified as "others". 
still unknown. Treponema may be associated with the reduction of ferric iron oxides, which has also been observed in ironreducing environments. ${ }^{28}$

It is noteworthy that the considerable enrichment of Thauera $(60 \%)$ in the SEM was observed compared with the SEC (12.9\%), indicating its important role in the conversion of propionate to methane. Thauera was also identified during the syntrophic oxidation of acetate and ethanol in paddy soil, ${ }^{13,21}$ and has been described for its ability to reduce Fe(III) oxides using acetate and ethanol as electric donors under anaerobic conditions. ${ }^{\mathbf{1 3 , 2 1 , 2 9}}$ Thus, a high proportion of Thauera species identified in the SEM suggested that it was most closely related to reduction of $\mathrm{Fe}(\mathrm{III})$ in magnetite. Additionally, 16S rRNA-based Illumina sequencing of the archaeal community in the SEM revealed that Methanobacterium (11\%) was the major dominant species, which utilizes $\mathrm{H}_{2}$ and $\mathrm{CO}_{2}$ to produce methane. ${ }^{21}$ These findings, combined with the finding showing that the degradation of propionate was concomitant with $\mathrm{H}_{2}$ production and $\mathrm{Fe}$ (II) formation, indicated that magnetite acted as an electron acceptor and may accelerate the rapid oxidation of propionate by the Fe(III)-reducing Thauera species in the SEM and facilitate the establishment of a syntrophic interaction of Thauera with Methanobacterium in a similar manner to ethanol degradation. ${ }^{21}$ Our finding showed a clear difference to previous reports, which showed that magnetite can act as a conduit of electrons to facilitate methane production in paddy soil with a significant enrichment in Geobacter and Methanosarcina ${ }^{\mathbf{9}, 11}$ and can accelerate methanogenesis from propionate by a methanogenic sludge. ${ }^{12}$ This difference could be ascribed to the difference in the microbial community structure between the previous findings and this work. Thus, the findings from this work demonstrated that direct IET might not substantially contribute to accelerated methane production.

\subsection{Degradation of the mixed VFAs}

The methane production performance of the artificial medium was investigated. The profiles of methane production during RBC are shown in Fig. 4. The data for methane production rate and acid removal are presented in Table 2. As shown in Fig. 4 and Table 2, increasing the cycle time led to a significant decrease in the methane yield and production rate in the SEC. The methane yield and production rate in cycle 3 were $57 \%$ and $60 \%$ lower than in cycle 1 . However, the methane yield and production rate in the SEM showed a significant increase with increasing cycle time. Firstly, the methane yield in cycle 3 was $55 \%$ higher than in cycle 1 . Secondly, the methane production rate in the cycle 1 was $0.119 \mathrm{mmol} \mathrm{d}^{-1}$, which was $84 \%$ lower than in cycle 3 .

Table 2 also showed that the methane production rate (average $0.16 \mathrm{mmol} \mathrm{d}^{-1}$ ) in the SEM was on average 3.6-fold higher than that (average $0.044 \mathrm{mmol} \mathrm{d}^{-1}$ ) in the SEC during RBC. The methane yield in the SEM was also higher than that in the corresponding SEC at each cycle of cultivation. These results indicated that the SEM can accelerate methane production from the mixed VFAs. When compared with previous paddy soil enrichments obtained in the presence of magnetite and

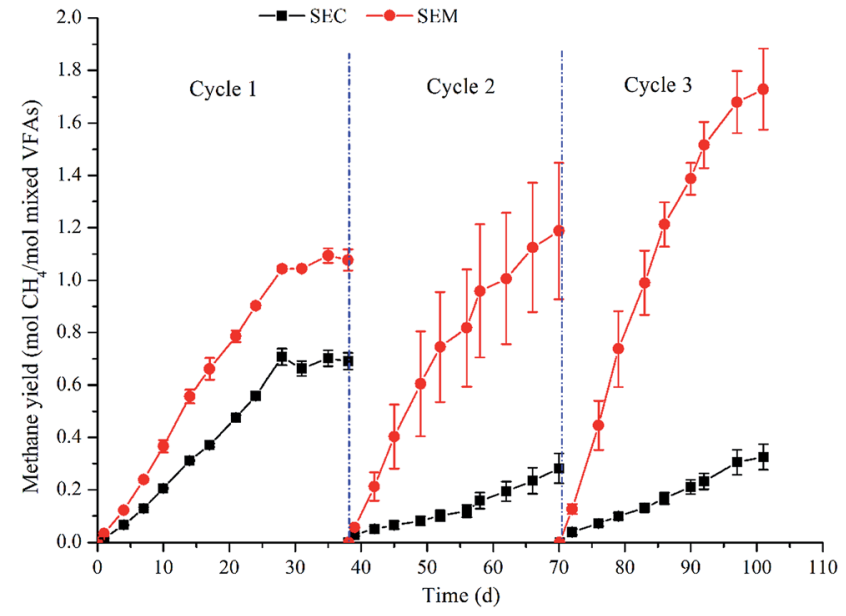

Fig. 4 The time course of methane production during RBC.

acetate,$^{13}$ the methane yield and production rate obtained in the SEM were respectively enhanced by $60 \%$ and $26 \%$ after two cycles of transfer. This further demonstrated that the SEM may act as a potential candidate for the rapid conversion of the mixed VFAs to methane. Further research is underway to test the potential of SEM to enhance the methane production performance of anaerobic digestion.

To explore why the methane production performance from the mixed VFAs in the SEM was significantly different from that in the SEC, the degradation of the individual VFAs was further analyzed. As shown in Fig. S8, $\dagger n$-butyrate, $n$-valerate, and $n$ caproate in the SEM showed a rapid degradation rate when compared with the SEC. Table 2 also shows that $n$-butyrate and $n$-caproate were nearly completely removed at the end of each feeding cycle in the SEM when compared with the SEC. The rapid degradation of these long-chain VFAs might also occur in a similar manner to the propionate degradation in the presence of magnetite as discussed in the preceding section. These findings indicated the potential excellent performance of SEM in the degradation of long-chain VFAs. However, although propionate was degraded in the SEM, compared with the SEC at the end stage of incubation (Fig. S8 $\dagger$ ), considerable amounts of acetate and propionate were accumulated in both the SEM and SEC at each cycle (Fig. S8 $\uparrow$ and Table 2). Comparative analysis showed that the accumulated acetate and propionate were produced from the degradation of $n$-butyrate, $n$-valerate and $n$ caproate according to the reactions listed in Table S3. $\dagger$ The accumulated acetate might interfere with propionate degradation. A previous report showed that the inhibition of propionate degradation by acetate occurred when the acetate concentration was over $23 \mathrm{mM}^{30}$ Thus, the low propionate degradation efficiency may be attributed to the accumulation of acetate in this work.

The results presented here showed that the SEM can rapidly convert the mixed VFAs to methane, indicating the remarkable application potential in enhancing the methane production rate of VFAs. 
Table 2 Comparison of methane production rate and fatty acid degradation during RBC

\begin{tabular}{|c|c|c|c|c|c|}
\hline & $R_{\mathrm{m}}\left(\mathrm{mmol} \mathrm{d}^{-1}\right)$ & Acetate (mM) & Propionate (mM) & n-Butyrate (mM) & n-Caproate (mM) \\
\hline \multicolumn{6}{|l|}{ SEC } \\
\hline Cycle 1 & 0.075 & $27.56 \pm 1.10$ & $16.15 \pm 0.31$ & $2.00 \pm 0.27$ & $2.41 \pm 0.26$ \\
\hline Cycle 2 & 0.028 & $38.29 \pm 0.73$ & $24.17 \pm 0.10$ & $11.64 \pm 1.00$ & $13.02 \pm 1.38$ \\
\hline Cycle 3 & 0.030 & $38.05 \pm 2.48$ & $30.31 \pm 0.21$ & $17.27 \pm 0.82$ & $15.43 \pm 0.60$ \\
\hline \multicolumn{6}{|l|}{ SEM } \\
\hline Cycle 1 & 0.119 & $31.95 \pm 1.95$ & $11.67 \pm 1.04$ & $0.18 \pm 0.09$ & $0.17 \pm 0.09$ \\
\hline Cycle 2 & 0.140 & $49.15 \pm 2.56$ & $22.92 \pm 0.83$ & $2.09 \pm 0.55$ & $2.07 \pm 0.43$ \\
\hline Cycle 3 & 0.219 & $22.80 \pm 10.73$ & $37.5 \pm 0.42$ & $0.09 \pm 0.03$ & $0.78 \pm 0.17$ \\
\hline
\end{tabular}

\section{Conclusions}

Magnetite nanoparticles added to paddy soil promoted methane production from propionate in a dose-independent manner concomitant with a specific enrichment of an $\mathrm{Fe}(\mathrm{III})$-reducing Thauera species. Results from inhibition experiments and DNA sequencing analysis showed that magnetite acted as an electron acceptor, may accelerate the rapid oxidation of propionate and facilitated the establishment of a syntrophic interaction of Thauera with Methanobacterium. Although the high concentrations of propionate and acetate have an adverse effect on methane production, SEM enabled a rapid conversion of the mixed VFAs to methane, demonstrating the practical application potential in enhancing the methane production performance of VFAs.

\section{Abbreviations}

\author{
VFAs Volatile fatty acids \\ IET Interspecies electron transfer \\ SEM Paddy soil enrichments obtained in the presence of \\ magnetite and propionate \\ TS Total solid \\ SEC Paddy soil enrichments achieved without magnetite \\ addition \\ RBC Repeated batch cultivation \\ XRD X-ray diffraction \\ FTIR Fourier transform infrared \\ TCD Thermal conductivity detector \\ HPLC High performance liquid chromatography
}

\section{Acknowledgements}

The authors thank Associated Prof. Bucai Zhang (Jiangsu Agrianimal Husbandry Vocational College) for providing the paddy soil. This work was funded by the National Natural Science Foundation of China (No. 21307143), 863 Project (2011AA060905), Key Research \& Development Project of Shandong (No. 2015GSF117016).

\section{References}

1 D. Xing, N. Ren and B. E. Rittmann, Appl. Environ. Microbiol., 2008, 74, 1232-1239.
2 K. J. J. Steinbusch, E. Arvaniti, H. V. M. Hamelers and C. J. N. Buisman, Bioresour. Technol., 2009, 100, 3261-3267.

3 W.-M. Wu, M. K. Jain and J. G. Zeikus, Appl. Environ. Microbiol., 1994, 60, 2220-2226.

4 Y. Gan, Q. Qiu, P. Liu, J. Rui and Y. Lu, Appl. Environ. Microbiol., 2012, 78, 4923-4932.

5 Y. Wang, Y. Zhang, L. Meng, J. Wang and W. Zhang, Biomass Bioenergy, 2009, 33, 1131-1138.

6 J. R. Sieber, M. J. McInerney and R. P. Gunsalus, Annu. Rev. Microbiol., 2012, 66, 429-452.

7 B. Schink, Microbiol. Mol. Biol. Rev., 1997, 61, 262-280.

8 A. J. M. Stams and C. M. Plugge, Nat. Rev. Microbiol., 2009, 7, 568-577.

9 S. Kato, K. Hashimoto and K. Watanabe, Environ. Microbiol., 2012, 14, 1646-1654.

10 H. Li, J. Chang, P. Liu, L. Fu, D. Ding and Y. Lu, Environ. Microbiol., 2014, 2015(17), 1533-1547.

11 S. Zhou, J. Xu, G. Yang and L. Zhuang, FEMS Microbiol. Ecol., 2014, 88, 107-120.

12 C. Cruz Viggi, S. Rossetti, S. Fazi, P. Paiano, M. Majone and F. Aulenta, Environ. Sci. Technol., 2014, 48, 7536-7543.

13 Z. Yang, X. Xu, R. Guo, X. Fan and X. Zhao, Bioresour. Technol., 2015, 190, 132-139.

14 C. Yamada, S. Kato, Y. Ueno, M. Ishii and Y. Igarashi, J. Biosci. Bioeng., 2014, 2015(119), 678-682.

15 Y. S. Kang, S. Risbud, J. F. Rabolt and P. Stroeve, Chem. Mater., 1996, 8, 2209-2211.

16 Z. Yang, R. Guo, X. Xu, X. Fan and S. Luo, Int. J. Hydrogen Energy, 2011, 36, 3465-3470.

17 D. R. Lovley and E. J. P. Phillips, Appl. Environ. Microbiol., 1987, 53, 1536-1540.

18 J. A. Peiffer, A. Sporb, O. Korenb, Z. Jin, S. G. Tringed and J. L. Dangle, Proc. Natl. Acad. Sci. U. S. A., 2013, 110, 65486553.

19 S. Fukuzaki, N. Nishio, M. Shobayashi and S. Nagai, Appl. Environ. Microbiol., 1990, 56, 719-723.

20 F. A. M. de Bok, A. J. M. Stams, C. Dijkema and D. R. Boone, Appl. Environ. Microbiol., 2001, 67(4), 1800-1804.

21 Z. Yang, X. Shi, C. Wang, L. Wang and R. Guo, Sci. Rep., 2015, $5,16118$.

22 S. Hattori, Microbes Environ., 2008, 23, 118-127.

23 B. Sun, J. R. Cole, R. A. Sanford and J. M. Tiedje, Appl. Environ. Microbiol., 2000, 66, 2408-2413. 
24 A. Ganesan, S. Chaussonnerie, A. Tarrade, C. Dauga, T. Bouchez, E. Pelletier, D. Le Paslier and A. Sghir, Int. J. Syst. Evol. Microbiol., 2008, 58, 2003-2012.

25 E. J. P. Jones, M. A. Voytek, M. D. Corum and W. H. Orem, Appl. Environ. Microbiol., 2010, 76, 7013-7022.

26 P. Liu, Q. Qiu and Y. Lu, Appl. Environ. Microbiol., 2011, 77, 3884-3887.

27 D. M. Akob, H. J. Mills, T. M. Gihring, L. Kerkhof, J. W. Stucki, A. S. Anastácio, K.-J. Chin, K. Küsel,
A. V. Palumbo, D. B. Watson and J. E. Kostka, Appl. Environ. Microbiol., 2008, 74, 3159-3170.

28 G. Baek, J. Kim, S. G. Shin and C. Lee, Appl. Microbiol. Biotechnol., 2016, 100, 927-937.

29 C. Ma, Z. Yu, Q. Lu, L. Zhuang and S.-G. Zhou, Appl. Microbiol. Biotechnol., 2015, 99, 3619-3628.

30 Q. Wang, M. Kuninobu, H. I. Ogawa and Y. Kato, Biomass Bioenergy, 1999, 16, 407-416. 